Revue Sciences/Lettres

Sciences / Lettres

4 | 2016

Baba Yaga en chair et en os

\title{
Baba Yaga dans les louboks
}

\section{Galina Kabakova}

\section{(2) OpenEdition}

Journals

Édition électronique

URL : http://journals.openedition.org/rsl/1000

DOI : $10.4000 /$ rsl. 1000

ISSN : 2271-6246

\section{Éditeur}

Éditions Rue d'Ulm

\section{Référence électronique}

Galina Kabakova, «Baba Yaga dans les louboks », Revue Sciences/Lettres [En ligne], 4 | 2016, mis en ligne le 16 janvier 2016, consulté le 19 avril 2019. URL : http://journals.openedition.org/rsl/1000 ; DOI : $10.4000 /$ rsl. 1000

Ce document a été généré automatiquement le 19 avril 2019

(c) Revue Sciences/Lettres 


\title{
Baba Yaga dans les louboks
}

\author{
Galina Kabakova
}

1 Le personnage de Baba Yaga attire l'attention des chercheurs depuis les premières publications de contes. Plusieurs hypothèses ont été formulées quant à l'origine du personnage et de son symbolisme. Une autre question qui a été longuement débattue concerne les fonctions du personnage. Est-il en rapport avec l'initiation, comme le pensait Vladimir Propp, avec la mort ou avec la naissance ? Symbolise-t-il la terre ou la fertilité ? Ces réflexions s'appuient toutes sur l'analyse de contes de tradition orale. Mais que nous apprend donc l'iconographie populaire de Baba Yaga ? L'étude d'images qui suit se propose de découvrir de nouvelles pistes de réflexion sur les origines du personnage, à partir d'un matériau visuel très original, celui des louboks russes du XVIII ${ }^{e}$ et du XIX ${ }^{e}$ siècle.

\section{Baba Yaga et ses compagnons}

2 Deux planches représentant Baba Yaga figurent dans toutes les études consacrées à l'imagerie populaire. La première s'intitule «Yaga-baba part combattre le crocodile » et la deuxième "Yaga Baba avec un moujik, un vieux chauve, sautent en dansant ». Les deux datent des années 1760 et apparaissent dans le catalogue de Dimitri Rovinski qui fait référence en la matière ${ }^{1}$.

3 La première image est connue dans deux versions, qui diffèrent légèrement par les motifs et par la légende. La légende complète de la première version explique : «Yaga-Baba part combattre le crocodile à dos de cochon avec un pilon. Ils ont une fiole de vin sous le buisson ». 
Image 1 - Yaga-Baba part combattre le crocodile à dos de cochon avec un pilon. Ils ont une fiole de vin sous le buisson (1760 environ).

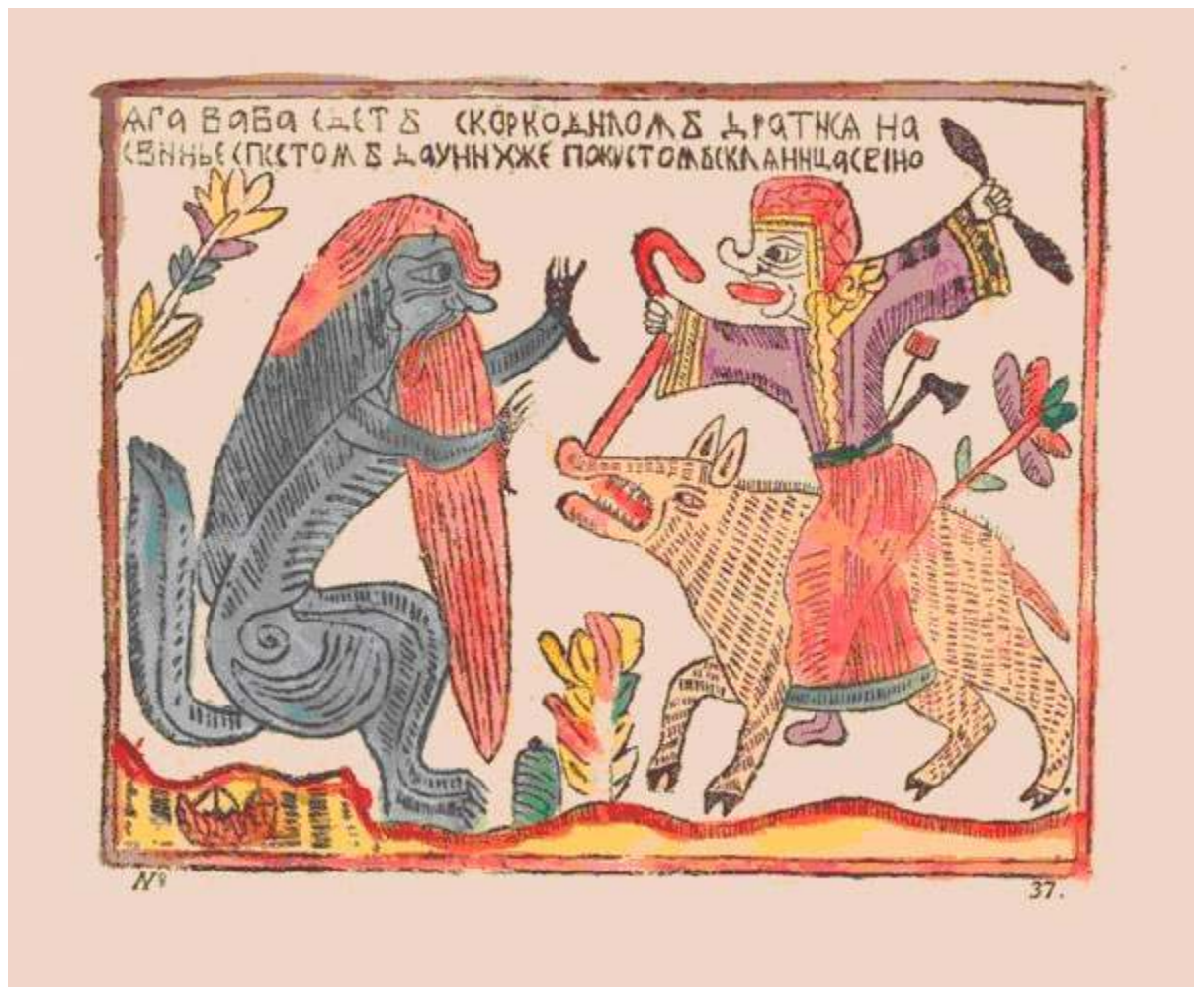

4 La deuxième version a une légende différente : «Baba Yaga jambe de bois part combattre à dos de cochon le karkadil avec son pilon. Il y a du vin ». 
Image 2 - Baba Yaga jambe de bois part combattre à dos de cochon le karkadil avec son pilon. Il y a du vin (1760 environ).

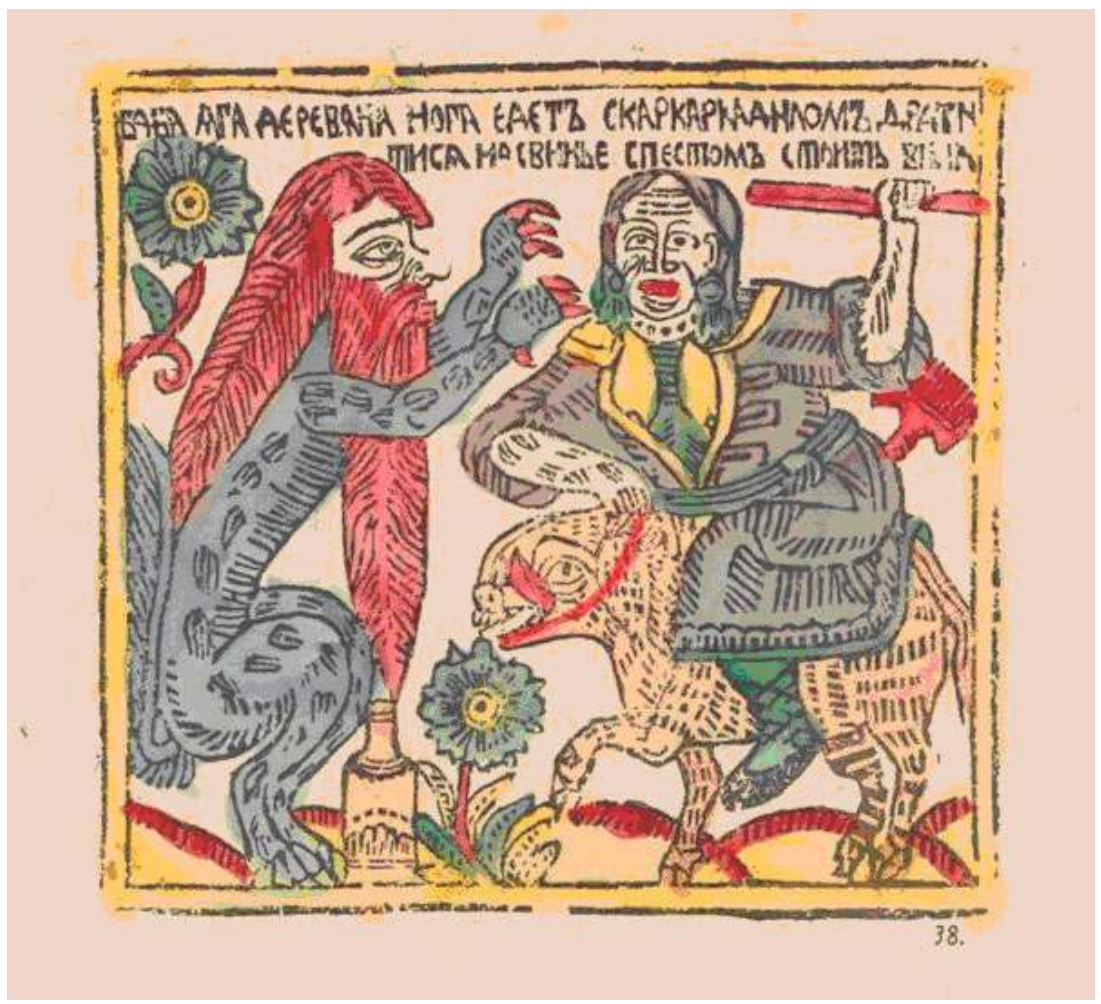

5 Les images sont intéressantes parce qu'elles représentent un personnage inconnu du folklore : le crocodile.

6 L'ouvrage récent de Konstantine Bogdanov, Crocodiles en Russie ${ }^{2}$, traite de la place de cet animal dans l'imaginaire russe en insistant sur ses connotations exotiques. Ce korkodil ou karkodil apparaît dans les bestiaires médiévaux et symbolise soit le diable, soit un homme hypocrite, d'où l'expression les «larmes de crocodile ». En même temps, l'iconographie des bestiaires est plus proche du crocodile réel. Dans les louboks, nous avons affaire à un animal fantastique composé de plusieurs bêtes avec des pattes et une crinière de lion, une queue de loup, des griffes et une barbe qui fait plutôt penser à la représentation du diable dans les livres manuscrits.

Outre ces deux versions de la première représentation célèbre de la Yaga avec le crocodile, on trouve une seconde image dans l'iconographie populaire, celle avec le Moujik. 


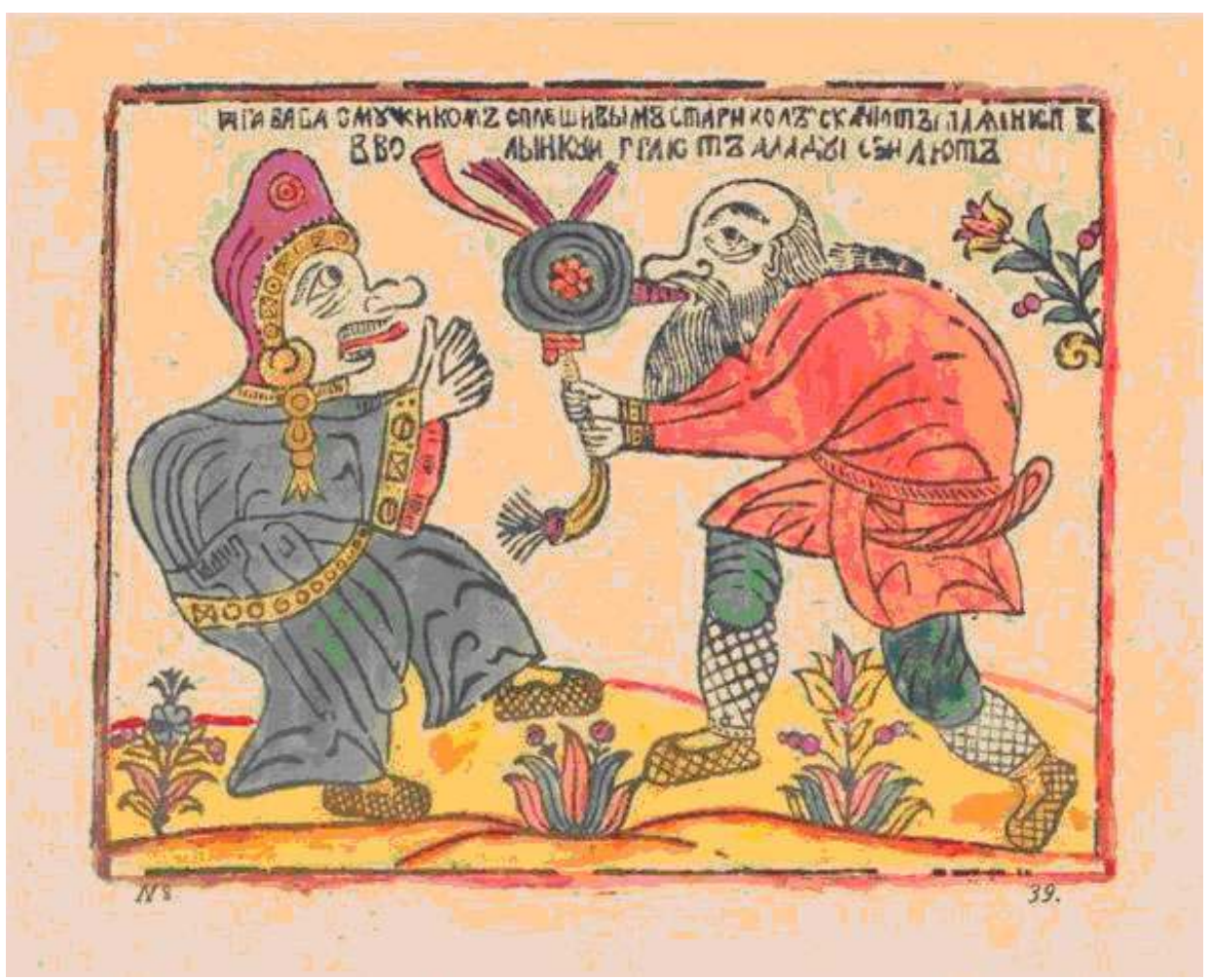

Cet autre compagnon de Baba Yaga, le moujik avec la cornemuse de la deuxième représentation, est habillé comme un vrai paysan avec ses chaussures russes tressées, lapti. La calvitie doit indiquer son âge avancé. La représentation de Baba Yaga varie d'une image à l'autre et fait montre de contradictions assez spectaculaires. Dans tous les cas, elle ressemble assez peu aux portraits de la sorcière brossés dans les contes. La fameuse unique jambe d'os n'apparaît pas. Elle a l'air d'une femme mais munie de traits hypertrophiés. Ainsi, son nez «en patate » dans les louboks 1 et 3, est recourbé vers le haut, et en même temps busqué (3). Les trois images mettent en évidence son énorme langue qui sort de la bouche. Et pour compléter ce portrait effrayant, la Baba Yaga dansante est bossue.

Dans cette série de gravures, elle est habillée comme les femmes russes aisées aux XvI ${ }^{\mathrm{e}}$ et XVII ${ }^{\mathrm{e}}$ siècle. L'habit qu'elle porte peut être identifié au letnik dont les manches et la ceinture sont peut-être brodées. Elle porte également une boucle et un collier, ce qui doit signaler son haut statut. Le bonnet dont elle est coiffée $\left(n^{\circ} 3\right)$ est celui d'une femme mariée ou d'une veuve. L'image $n^{\circ} 2$ au contraire la présente les cheveux défaits, ce qui à l'époque était considéré comme indécent.

La Baba Yaga des images 2 et 3 porte des lapti comme le moujik paysan, ce qui crée une certaine dissonance par rapport à son habit, celui d'une femme noble ou, du moins, riche. L'habit de la première Baba Yaga est différent car il ressemble beaucoup à l'habit d'une femme finnoise avec son bonnet caractéristique.

11 Tout aussi intéressants sont les attributs de ces Babas Yaga. Le mortier ou le balai qui lui servent de moyen de locomotion dans les contes sont absents et elle avance fièrement perchée sur un cochon ou un sanglier. Dans les deux premières images elle est munie à la 
fois d'ustensiles féminins, comme le peigne, et d'ustensiles masculins comme la hache. On reconnaît également les rênes et le pilon dont elle aiguillonne le cochon.

D'ailleurs, le pilon en tant qu'arme se retrouve dans d'autres louboks. Il sert à la fois à Baba Yaga dans les contes - dans son mortier, elle s'approche d'un vieux et le frappe avec son pilon («Fedor Vodovitch et Ivan Vodovitch $\left.{ }^{3} »\right)$ - et à la femme méchante dans le loubok. Ainsi, dans une version de livre manuscrit du XviII ${ }^{\mathrm{e}}$ siècle on trouve une image populaire qui représente l'épouse méchante qui chasse son mari de la maison en tenant dans une main une cruche et dans l'autre un pilon'

Le couple énigmatique composé du crocodile et de Baba Yaga dispose d'autres attributs intéressants : ce sont la bouteille de vin posée devant le crocodile ainsi que le petit bateau qu'on reconnaît dans l'eau en-dessous du crocodile (1). Le paysage symbolique est composé également de fleurs et de branches pour signaler que la nature est en fleurs.

Cette présentation intrigue les universitaires depuis plus d'un siècle. En effet, si Baba Yaga ressemble si peu à son prototype merveilleux, ne s'agit-il pas d'un tout autre personnage qui se cache derrière le nom de la célèbre sorcière?

Dimitri Rovinski a été le premier à supposer qu'il s'agissait d'une satire politique visant le premier empereur russe Pierre le Grand et son épouse ${ }^{5}$. En effet, le bateau pourrait symboliser la passion du tsar pour la marine qui l'avait amené à construire une nouvelle capitale maritime, Saint-Pétersbourg. Le bonnet et le costume finnois de la femme seraient alors la référence à l'origine balte de sa deuxième épouse qui deviendra l'impératrice Catherine $\mathrm{I}^{\mathrm{re}}$. Le vin indiquerait leur penchant commun pour l'alcool et la posture martiale de la femme évoquerait les relations tumultueuses du couple. Mais qui pourrait donc ironiser ainsi sur le couple impérial? Rovinski a supposé que les gravures étaient produites par des vieux-croyants qui nourrissaient une haine tenace pour leur ennemi juré et l'affublaient de sobriquets comme «chat» et "crocodile». D'autres images populaires pourraient également être interprétées comme une satire contre Pierre le Grand, comme par exemple celle où il est représenté comme le chat : « Le Chat de Kazan ou Les obsèques du chat par les souris ».

Cette interprétation a été systématiquement répétée par plusieurs chercheurs jusqu'à nos jours. Mais elle suscite également des interrogations. Que signifie notamment la longue barbe du crocodile si on prend en compte le fait que Pierre avait déclaré la guerre aux barbes $^{6}$ ? De plus les gravures dont on dispose datent des années 1760 et on n'a pas de preuves que les originaux aient été réalisés un demi-siècle plus tôt. Diane Farrell dans sa thèse Popular prints in the cultural history of eighteenth-century Russia a émis des doutes quant au contexte politique de ces images. La chercheuse américaine suppose notamment qu'il s'agit plutôt de personnages carnavalesques ${ }^{7}$. Le dernier en date, Konstantin Bogdanov, insiste sur le caractère purement divertissant de ces images qui s'inscrivent bien dans la lignée d'autres scènes comiques représentant des ivrognes et des bagarreurs ${ }^{8}$ . On pourrait ajouter que ces trois gravures s'insèrent également dans la thématique des images antiféministes, voire misogynes, où les femmes, surtout les jeunes épouses, cherchent à humilier et à malmener leurs maris. Parfois cette satire vise plus précisément les femmes étrangères. 


\section{En quête des origines de Baba Yaga}

17 En préparant l'anthologie de contes étiologiques russes publiée en 2005 sous le titre Contes et légendes de Russie, j'ai trouvé un récit assez court enregistré dans l'est de la Russie, dans la vallée de Kama, région qui se singularise par la très forte présence des vieux-croyants.

Un diable eut envie de fabriquer Baba Yaga. Il rassembla les douze bonnes femmes les plus méchantes et les mit à cuire dans un chaudron. Il goûta, puis fit cuire encore un jour. Il goûta de nouveau et éternua si fort que les portes s'ouvrirent. Il mangea une cuillerée de la préparation et cracha. Du chaudron sortit alors Baba Yaga'.

Malgré le fait que le texte figure dans l'Index des contes-types du conte des Slaves orientaux sous le numéro SUS $1169^{*}$, il reste isolé car aucune autre version n'a été enregistrée ni en Russie, ni en Ukraine, ni en Biélorussie. C'est après avoir publié ce texte que j'ai découvert une planche de loubok composée de huit images, huit séquences qui racontent la création de Baba Yaga. Le conte cité ci-dessus représente la version abrégée du début du récit figurant sur le loubok en huit planches.

Image 4 - les origines de Baba Yaga.

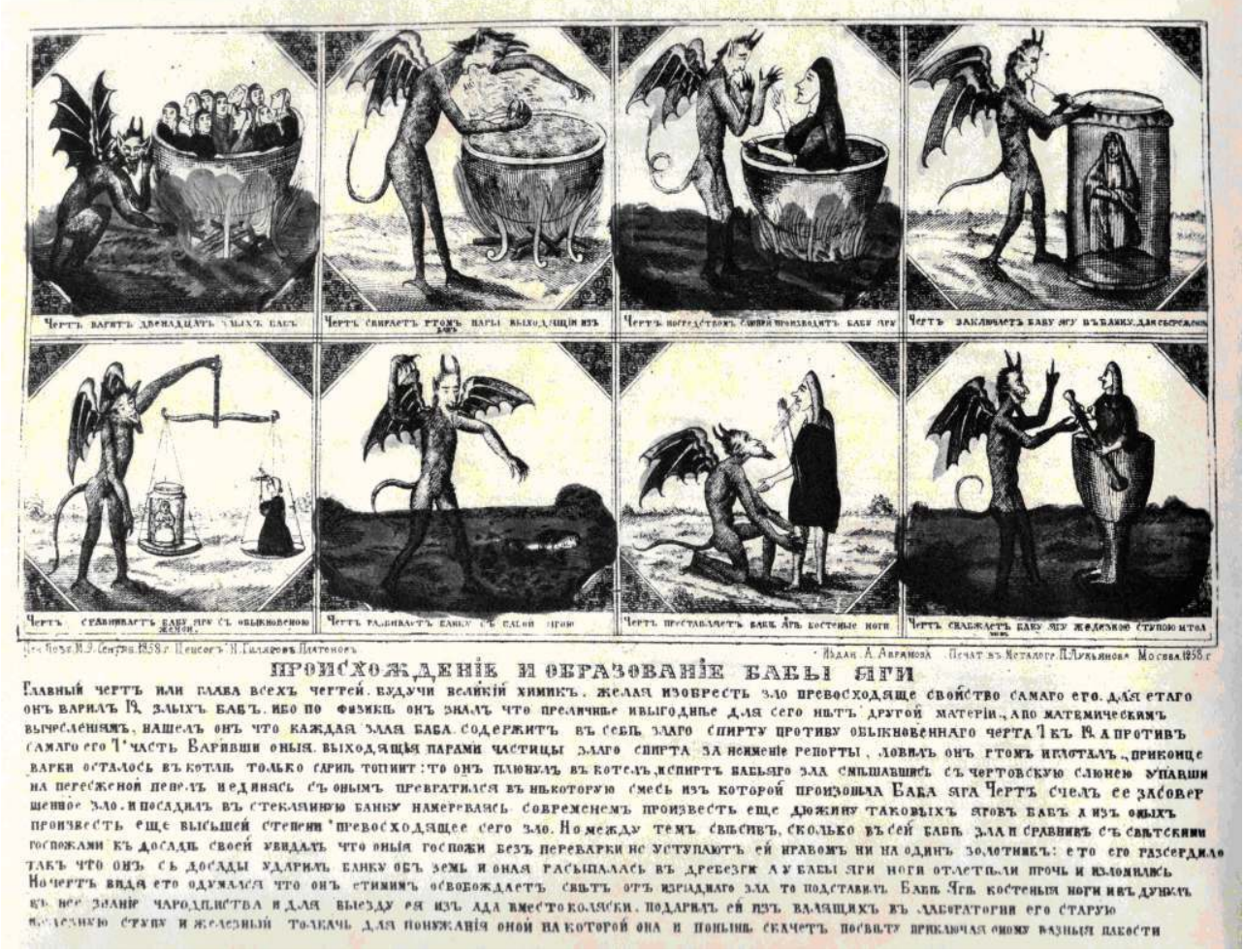

C'est un loubok assez rare qui ne figure pas dans le catalogue de l'image populaire de Dimitri Rovinski. Un exemplaire est conservé au Musée d'histoire de la religion à SaintPétersbourg, un autre à la Bibliothèque nationale à Saint-Pétersbourg, enfin un troisième à la Bibliothèque nationale de France. C'est ce dernier qui a été reproduit dans la monographie de Catherine Claudon-Adhémar, Imagerie populaire russe (Milan, 1977). Voici donc le texte complet de la légende. 
Origine et formation de Baba Yaga

Le diable en chef ou le chef de tous les diables, tout en étant grand chimiste, eut envie d'inventer le mal qui le dépasserait par sa puissance. Dans ce but, il cuisit douze femmes méchantes, car la physique lui avait apprit que c'était la meilleure et la plus profitable des matières. Et d'après les calculs mathématiques chaque femme méchante contient du mauvais alcool en considérant un diable normal dans la proportion de sept contre douze et contre lui en personne une septième. En faisant cuire ces particules du mauvais alcool qui se dégageaient avec la vapeur en l'absence de la cornue il les attrapait avec la bouche et les avalait. En fin de cuisson il ne restait dans le chaudron que la matière brûlée ; il cracha dans le chaudron et l'alcool du mal de femmes mêlé à la salive du diable tombée sur les cendres, en fusionnant, forma Baba Yaga. Le diable la considéra comme le mal absolu et la plaça dans un bocal dans l'idée de fabriquer par la suite une dizaine de Baba Yaga comme celle-ci et en fabriquer ensuite le mal encore plus parfait. Mais en pesant le mal qu'elle contenait et le comparant au mal propre aux femmes du monde, il constata, désappointé, que ces dames même sans cuisson ne lui cèdent pas une once. Cela le fâcha au point que, par dépit, il jeta le bocal par terre qui se brisa en mille morceaux, comme les jambes de Baba Yaga qui, arrachées, s'étaient cassées. Cela dégrisa le diable qui, conscient qu'il libérerait ainsi le monde du grand mal, donna à Baba Yaga des jambes en os, lui inspira la connaissance de la sorcellerie et pour qu'elle quitte l'enfer lui offrit en guise de voiture un vieux mortier en fer qui traînait dans son laboratoire et un pilon en fer pour aiguillonner le mortier qui lui sert toujours à parcourir le monde en faisant le mal.

C'est un texte assurément satirique qui insiste sur le caractère "scientifique » de la fabrication de Baba Yaga qui fait appel à la fois aux mathématiques, à la chimie et à la physique. Mais il est clair que le message de cette planche satirique vise avant tout la méchanceté des femmes. Pour une fois la satire ne vise pas toutes les femmes méchantes, comme ça se faisait depuis le Moyen Âge, mais les femmes de la haute société.

Cette satire peut se nourrir des modèles occidentaux : rappelons la célèbre série française de Lustucru où ce forgeron cherche à remodeler les femmes précieuses et même l'alambic miraculeux qui permet de refaire les femmes. Le thème de la fabrication n'est pas sans rappeler le motif de l'homoncule créé par un alchimiste moyenâgeux. Le motif de la pesée de Baba Yaga et d'une mondaine fait référence à un thème traité par les icônes : «Saint Michel et Satan qui pèsent les âmes de pécheurs ».

En même temps, on reconnaît quelques motifs propres aux récits étiologiques, comme la salive qui aide à fabriquer un personnage humain (en général, c'est la salive divine qui permet de fabriquer des figurines en argile qui deviendront les premiers hommes). Dans la tradition orale, on trouve également des récits qui présentent le diable en tant que créateur : par exemple, il arrive à fabriquer les différentes nations. Il s'agit d'un conte ukrainien et le mode opératoire n'est pas sans rappeler notre planche : le diable met dans un chaudron des herbes et de la poix et les fait cuire. Selon le temps de cuisson, il en sort d'abord un Ukrainien, un Allemand, un Tatar et un Juif, le dernier est considéré par le diable comme le plus réussi ${ }^{10}$. Même si ce récit est prétendument étiologique, on y trouve quand même la caractéristique permanente du diable : c'est un créateur malheureux ; il essaie de créer l'homme mais il échoue, dans d'autres cas il cherche à imiter l'œuvre divine et il crée tout autre chose. Et dans notre cas, il essaie de fabriquer quelque chose d'inédit qui dépasserait par sa nuisance tous les êtres existants et finalement il ne fait que reproduire les méchantes femmes du monde.

23 La naïveté du dessin fait penser que l'auteur n'a pas copié une gravure occidentale (contrairement à d'autres louboks consacrés à cette problématique, comme par exemple 
celui qui représente un couple en train de s'affronter pour savoir qui va porter la culotte). Le graveur est assez malhabile dans le traitement des personnages. Le diable ressemble à un homme, mais muni des détails obligatoires pour un diable russe: une queue, des cornes et une barbichette de bouc, des ergots de coq, des griffes mais aussi des ailes pour rappeler ses origines célestes (d'ailleurs, ce dernier détail n'est pas systématique dans la représentation de Satan). La grande similitude entre le portrait du diable, des femmes et de Baba Yaga, produit final, serait-elle voulue (comme par exemple dans la gravure française illustrant la vraie nature de la gent féminine, "La vraye femme ») ? Je pense qu'elle résulte plutôt de la maladresse du graveur que de son dessein. Il est aussi intéressant de souligner la représentation banalisée de Baba Yaga et des femmes mondaines qui ne portent aucun signe distinctif de leur statut, aucune trace de la coquetterie. En fait, les seuls détails qui constituent le portrait de la méchante sorcière sont ses deux jambes (au lieu d'une dans le folklore) en os et son mortier avec le pilon qui lui servent de moyen de locomotion.

Les recherches de la source de cette planche si mystérieuse m'ont conduite à la littérature du colportage qui a fait la part belle aux adaptations et à la réécriture des contes traditionnels. Cette littérature « du loubok » a précédé la publication «scientifique » de contes au XIX ${ }^{e}$ siècle; Alexandre Afanassiev a en partie puisé dans ces éditions de la deuxième moitié du XVIII ${ }^{\mathrm{e}}$ siècle lorsqu'il considérait les contes proches de leur source populaire.

C'est ainsi que j'ai retrouvé le texte du loubok dans le "Conte du gentilhomme Zaolechanine, preux au service de prince Vladimir » qui fait partie des "Contes russes » en 10 volumes de Vassili Levchine (1780). Ce texte, à la différence de celui du loubok, cite le terme alchimique caput mortuum [le brun momie] qui permet de créer la pire des créatures, Baba Yaga :

De l'origine de Baba Yaga

Le diable en chef ou le diable au-dessus de tous les diables, tout en étant grand chimiste, cuisit douze femmes méchantes, en espérant d'en extraire l'essence du mal qui le dépasserait par sa qualité. La physique lui avait appris que c'était la meilleure et la plus profitable des matières, et d'après les calculs mathématiques chaque femme méchante contient du mauvais alcool en considérant un diable normal dans la proportion de 7 contre 22 et contre lui en personne une $11^{\mathrm{e}}$, c'est pourquoi il en cuisait 12. Mais comme la cornue n'était pas encore inventée, il attrapait avec la bouche les particules de l'alcool qui se dégageaient avec la vapeur. En fin de cuisson il ne restait dans le chaudron que du caput mortuum; dissipé, il cracha dans le chaudron; l'alcool mêlé à la salive du diable tomba sur ce caput mortuum, et le diable, dépassé dans ses attentes, découvrit Baba Yaga qui en sortit.

Dans le conte de Levchine, ce texte légende un portrait dessiné sur un mur du château ensorcelé. Et il débouche sur la mise en garde :

Cette nouvelle t'est communiquée, ô lecteur curieux, de la part d'une personne qui protège ton sauveur. Mais comme il est convenu que le mystère dévoilé contre le gré d'une femme soit puni, sois métamorphosé pour ta désobéissance à Baba Yaga.

Et en effet, le personnage qui raconte la découverte est transformé en un clin d'œil en dragon $^{11}$. 


\section{Baba Yaga dans la littérature «de loubok»} et d'autres sont ajoutés. Voyons par exemple le motif du cannibalisme et prenons l'exemple du conte-type ATU 327C, "Le diable apporte chez lui l'enfant dans le sac». Dans la version russe, il s'agit toujours de la sorcière Baba Yaga qui attrape un enfant et essaie de le cuire au four. Dans les versions populaires du conte russe, comme dans «Hansel et Gretel » des frères Grimm, c'est la sorcière ou sa fille ou bien les deux qui périssent dans le feu enfournées par l'enfant rusé. Il en va autrement dans la littérature du loubok. Ainsi dans le "Conte du gentilhomme Zaolechanine", la sorcière réussit à capturer un enfant de six ans, à le rôtir et le manger ${ }^{12}$.

jonction entre Baba Yaga et le dragon, qui est l'antagoniste attitré du héros, peut prendre une tournure différente de la tradition orale: dans le « Deuxième conte d'Ivan le Tsarévitch » du recueil Contes russes de Piotr Timofeev (1787) le roi dragon Erakski qui avait capturé l'héroïne Maria Morevna part au combat contre Baba Yaga, ce qui permet à Ivan le Tsarévitch de la libérer sans problème. Rappelons qu'en règle générale, c'est au héros qu'incombe la tache d'affronter et d'occire le méchant dragon.

De même, dans « Le conte de Léviane le preux » du recueil anonyme Le Narrateur de contes russes (1787) ce n'est pas le héros qui est mandaté pour garder les pouliches fabuleuses de Baba Yaga, comme c'est le cas dans les contes populaires «Le cheval magique » (ATU 302C), mais Kachtchej l'Immortel, un autre antagoniste redoutable.

Dans le "Conte du gentilhomme Zaolechanine », on découvre également des thèmes «romanesques» inventés par l'auteur: Baba Yaga adopte l'héroïne, elle éprouve de l'amour pour le dragon ailé, qui ne le partage pas. Néanmoins « elle lui rend visite tous les jours et rentre parfois en larmes et parfois en grande colère qui se solde toujours par des soupirs ». Et pour finir, quand Baba Yaga décède, «son âme mesquine quitte son siège misérable et chute en enfer ${ }^{13}$ ».

cest surtout dans les descriptions que l'on découvre des détails absents des versions orales. Ainsi, dans le «Deuxième conte d'Ivan le Tsarévitch", la sorcière vit dans un palais protégé par une barrière en fer et non plus dans une cabane perchée sur des pattes de poulet.

ans une version anonyme de "L'oiseau de feu et le cheval à la crinière d'or " publiée à Moscou en 1860, on fournit une image composite de notre sorcière. Elle a « une tête de porc, une queue de corbeau ou une queue en os » ou bien " elle a deux cornes, une tête de chien, un nez d'oie, une queue d'étain ». Ou encore on évoque "l'affreuse Baba Yaga à longues dents, jambe d'os, tête de fonte, queue d'argile ${ }^{14}$ ».

Rappelons à ce propos que le personnage folklorique de Baba Yaga est décrit de manière assez succincte et ce ne sont pas les mêmes caractéristiques physiques qui sont mises en avant. On insiste sur les traits qui la rapprochent du monde des morts ou des reptiles: elle ne dispose que d'une jambe et celle-ci est en os, autrement dit, décharnée. Et son nez est parfois si long qu'il atteint le plafond. Parfois on mentionne des traits qui suggèrent sa féminité hypertrophiée: des seins si énormes qu'ils ne tiennent pas dans la pièce et débordent par-dessus du seuil. Et on évoque aussi les détails composites comme le nez de fer ou la face d'argile ${ }^{15}$. 
Pour conclure cette étude iconographique des images et légendes gravées sur bois, on peut constater que le loubok se saisit du personnage des contes, de grande notoriété dans la tradition orale, non pour en raconter l'histoire, mais essentiellement dans le but de dénigrer les faiblesses de la gent féminine en général, en se focalisant soit sur les femmes du monde, à la réputation d'être méchantes, soit sur les femmes étrangères. L'imagerie populaire montre ainsi les préjugés à la fois sociaux, de genre et de race ayant cours à l'époque.

\section{NOTES}

1. Dm. Rovinskij, Russkie narodnye kartinki, vol. 1, Saint-Pétersbourg, 1900, p. 273-274.

2. K. Bogdanov, O krokodilax v Rossii, Moscou, NLO, 2006.

3. Severnye skazki v sobranii N.E. Ončukova, Saint-Pétersbourg, Mir, 2008, p. 84-89.

4. F. I. Buslaev, « Ženščina v narodnyx knigax », Moi dosugi, part. 2, Moscou, 1886, p. 44-45.

5. Dm. Rovinskij, op. cit., p. 273-274.

6. Pierre le Grand, lors des grands projets de modernisation de la Russie, avec notamment la construction de Saint-Pétersbourg, avait instauré de nombreux impôts et notamment un impôt sur le port de barbe. Cela était vu comme une persécution supplémentaire des vieux-croyants qui portaient la barbe.

7. D. Farrell, Popular prints in the cultural history of eighteenth-century Russia, thèse de doctorat, Madison, Université du Wisconsin, 1980, p. 96.

8. K. Bogdanov, op. cit., p. 184-187.

9. G. Kabakova, Contes et légendes de Russie, trad. A. Stroeva, Paris, Flies France, 2005, p. 188.

10. G. Kabakova, «La figure de l'étranger dans les contes étiologiques », in A. Stroev (dir.), L'Image de l'étranger, Paris, Institut d'études slaves, 2010, p. 347.

11. V. Levšin, Russkie skazki, livre 1, Saint-Pétersbourg, Tropa Trojanova, 2008, p. 363.

12. Ibid, p. 330.

13. Ibid, p. 330-333.

14. A. Orlova, Obraz Baby-Yagi v russkix volšebnyx skazočnyx povestjax XVIII veka (http: //msuresearch06.ru/index.php/filology/165-russustn/1140-2011-03-17-12-07-27 ?

tmpl =component\&print =1\&page =); K. Korepova, Russkaja lubočnaja skazka, Moscou, Forum, 2012, P. 141.

15. V. Dobrovol'skaja, «Baba Yaga: k voprosu ob iskonnoj prirode », Obraznyj mir tradicionnoj kul'tury, Moscou, GRCKF, 2010, P. 30. 


\section{RÉSUMÉS}

L'iconographie populaire de Baba Yaga n'est pas très abondante. Une image de loubok a attiré tout particulièrement mon attention. Il s'agit d'une planche peu connue, qui raconte la création de Baba Yaga. C'est un loubok assurément satirique qui vise avant tout la méchanceté des femmes. Nous procédons à l'analyse de différentes hypothèses émises quant à la signification d'autres images, plus célèbres: "Yaga-baba part combattre le crocodile», connue en deux versions, et « Yaga Baba avec un moujik ».

The popular iconography of Baba Yaga is not very abundant. An image from the Lubok tradition has particularly caught my attention. This is a little-known print that tells of the creation of Baba Yaga. This is certainly a satirical Lubok aimed primarily at the wickedness of women. I proceed to the analysis of different hypotheses about the meaning of other, more famous prints: « Baba Yaga rides off to fight the crocodile», printed in two versions, and "Yaga Baba with mujik », Baba Yaga with peasant.

\section{INDEX}

Mots-clés : contes populaires, légendes étiologiques, loubok

Keywords : folk and fairy tales, etiological legends

\section{AUTEUR}

\section{GALINA KABAKOVA}

Maître de conférences en civilisation russe, UFR d'études slaves, université Paris-Sorbonne. galina.kabakova@paris-sorbonne.fr Parmi les publications : Anthropologie du corps féminin dans le monde slave, Paris, Montréal, L'Harmattan, 2000 ; Le Corps dans la culture russe, Moscou, Novoe literaturnoe obozrenie, 2005 (en russe) ; L'Hospitalité, le repas, le mangeur dans la civilisation russe, Paris, L'Harmattan, 2013 ; Contes et légendes étiologiques dans l'espace européen, Paris, Pippa/Flies France, 2013 ; Les Traditions russes d'hospitalité et de convivialité, Moscou, Forum-Neolit, 2015 (en russe) ; ainsi que la publication de sept anthologies de contes et légendes étiologiques, dont Contes et légendes de Russie, Paris, Flies France, 2005 et Aux origines du monde : contes et légendes étiologiques russes, Moscou, Forum - Neolit, 2014 (en russe). 